Discussion Paper No. 915

\title{
PRODUCT DIFFERENTIATION AND ENTRY TIMING IN A CONTINUOUS TIME SPATIAL COMPETITION MODEL
}

\author{
Takeshi Ebina \\ Noriaki Matsushima \\ Daisuke Shimizu
}

October 2014

The Institute of Social and Economic Research

Osaka University

6-1 Mihogaoka, Ibaraki, Osaka 567-0047, Japan 


\title{
Product differentiation and entry timing in a continuous time spatial competition model*
}

\author{
Takeshi Ebina \\ Faculty of Economics, Shinshu University, \\ Noriaki Matsushima ${ }^{\ddagger}$ \\ Institute of Social and Economic Research, Osaka University \\ Daisuke Shimizu ${ }^{\S}$ \\ Faculty of Economics, Gakushuin University
}

October 20, 2014

\begin{abstract}
We extend the well-known spatial competition model (d'Aspremont et al., 1979) to a continuous time model in which two firms compete in each instance. Our focus is on the entry timing decisions of firms and their optimal locations. We demonstrate that the leader has an incentive to locate closer to the centre to delay the follower's entry, leading to a non-maximum differentiation outcome. We also investigate how exogenous parameters affect the leader's location and firms' values and, in particular, numerically show that the profit of the leader changes nonmonotonically with an increase in the transport cost parameter.
\end{abstract}

Keywords: Location; Hotelling model; Continuous time; Entry timing

JEL Code: L13, R32, C72

\footnotetext{
*The authors gratefully acknowledge financial support from a Grant-in-Aid for Young Scientists (24730224) and Basic Research $(24530248,24530264)$ from the MEXT and the Japan Society for the Promotion of Science. The second author thanks the warm hospitality at MOVE, Universitat Autònoma de Barcelona where part of this paper was written. Needless to say, we are responsible for any remaining errors.

${ }^{\dagger}$ Faculty of Economics, Shinshu University, 3-1-1, Asahi, Matsumoto, Nagano, Japan. Phone: (81)-48021-7663. Fax: (81)-4-8021-7654. E-mail: ebina@shinshu-u.ac.jp

${ }^{\ddagger}$ Institute of Social and Economic Research, Osaka University, Mihogaoka 6-1, Ibaraki, Osaka, Japan. Phone: (81)-6-6879-8571. Fax: (81)-6-6879-8583. E-mail: nmatsush@iser.osaka-u.ac.jp

${ }^{\S}$ Faculty of Economics, Gakushuin University, 1-5-1, Mejiro, Toshima-ku, Tokyo 171-8588, Japan. Phone: (81)-3-5992-3633. FAX: (81)-8-5992-1007. E-mail: daisuke.shimizu@gakushuin.ac.jp
} 


\section{Introduction}

Researchers in economics and marketing have emphasised the importance of (horizontal) product differentiation in the context of firm competition (e.g. d'Aspremont et al., 1979; Brown, 1989; Lancaster, 1990). When firms launch their new products into markets, timing and product characteristics are some of the important factors for their profits (e.g. Krishnan and Ulrich, 2001). Taking into account firms' decisions regarding product differentiation, researchers theoretically and/or empirically investigate how firms determine the timing of launching their products and those characteristics (e.g. Lambertini, 1997; Thomadsen, 2007).

From the theoretical point of view, Lambertini (2002) presented pioneering work that discusses the strategic interaction between the optimal locations of the inventor (the market leader), who anticipates subsequent entry and the location choice of the follower in a Hotelling-type spatial competition model, as in d'Aspremont et al. (1979). ${ }^{1}$ He was the first to introduce a dynamic model in the sense that time is continuous, the firm locations are fixed once entry is made and that firms earn their profits in each instance. Regarding the time structure, several papers deal with sequential locational entry in a discrete time model, which allows qualitative analyses such as how many steps the timing of investment would change given a change in other parameters (e.g. Prescott and Visscher, 1977). However, a more rigorous quantitative analysis, such as determining the percentage change in the investment time attributable to a percentage change in a parameter, requires a continuous time model. ${ }^{2}$

\footnotetext{
${ }^{1}$ Location point is interpreted as a firm's differentiation selection because the distance between a firm's location point and a consumer's address corresponds to that between a firm's attribute and a consumer's ideal point. This interpretation is standard in spatial economics and marketing literature.

${ }^{2}$ Continuous time models are often used in models such as real option game models that investigate the timing problem of firms' entry without the locational context (e.g. Dixit and Pindyck, 1994, Chapter 9; Azevedo and Paxson, 2014). These studies introduce one or more probabilistic fluctuations into their models. Our model is deterministic and does not focus on this randomness but instead, focuses on the relation between location and entry timing. The real option game approach is useful for taking into account the endogenous timing decision.
} 
This novel point is from Lambertini (2002) and differs significantly from those in related theoretical papers discussing sequential location choices based on Hotelling-type spatial competition models (e.g. Neven, 1987; Götz, 2005). ${ }^{3}$ Those related papers are static Hotelling models in the sense that each firm has only one profit earning chance. ${ }^{4}$ Lambertini (2002) considered two scenarios: (i) the follower's timing of entry is exogenous and (ii) the follower's timing of entry is probabilistically determined. Therefore, the follower does not endogenously determine its optimal timing of entry in either scenario. To summarise, Lambertini (2002) considered a continuous time model, but an endogenous entry timing model with continuous time has not been considered in locational models. Because the entry timing of followers significantly influences market leaders as well as followers (Kalyanaram et al., 1995; Vakratsas et al., 2003), we need to overcome the weakness in the model given by Lambertini (2002) and endogenise the follower's entrytiming decision. Therefore, our paper substantially extends the model of Lambertini (2002).

We incorporate several aspects into the standard Hotelling duopoly model in d'Aspremont et al. (1979). The time horizon is infinite, as in Lambertini (2002). Each firm sets a price and earns a profit in each instance if it exists in the market, implying that a delay of entry causes a loss of profit opportunity. In anticipation of subsequent entry by the follower, the market leader initially sets its location. Because the leader's location decision influences the profits of the follower, it also affects the timing of the entry (the length of the monopoly period), thus representing an additional value of our paper. After the location choice of the market leader, the follower determines the timing of entry and its location. When the follower enters the market, it incurs an investment cost that exponentially decreases with the standard discount rate. In contrast, consumer size increases with a growth rate lower than the discount rate. By balancing the benefit and cost of

\footnotetext{
${ }^{3}$ Many papers discussed sequential location choices in spatial competition models. Kress and Pesch (2012) and Biscaia and Mota (2013) provided comprehensive surveys on spatial competition.

${ }^{4}$ Lambertini (1997) and Meza and Tombak (2009) considered the endogenous timing of locations in such static Hotelling models.
} 
staying outside, the follower determines its entry timing and location.

Compared with Lambertini (2002), our contributions in this paper are threefold. The first contribution is that we endogenise the follower's timing. The second contribution follows the first, as we introduce investment costs and a growth rate in consumer size to make the model more realistic. In addition, the growth rate ensures that the entry occurs within a finite time ${ }^{5}$ and, in turn, affects the leader's location. The third contribution is a strategic interaction between the leader's location and the follower's entry timing. In addition to the effects considered by Lambertini (2002), the leader's moving closer to the centre increases the follower's incentive to delay its entry, prolonging the monopoly regime. Thus, by endogenising the follower's timing, the leader has a stronger incentive to move closer to the central point. Although this strategic interaction among the leader's location, the follower's location and its entry timing is an important aspect of this problem, Lambertini (2002) does not take into account this strategic interaction because of his assumption of an exogenous entry timing by the follower.

We also show that the follower always chooses to maximise the distance between the firms whereas the leader has an incentive to locate closer to the centre to delay the follower's entry, possibly leading to a non-maximum differentiation outcome. Furthermore, the location interval between the leader and the follower is negatively correlated with the length of time for which the follower stays outside. These results are similar to those in Lambertini (2002), although the mechanism behind these results definitely differs between the two papers.

Finally, we show that the entry timing becomes earlier as the growth rate of consumer size or the parameter of consumer transport cost increases, and becomes later as the discount rate increases. We numerically investigate how those exogenous parameters influence their profits. A notable result is that the profit of the market leader non-

\footnotetext{
${ }^{5}$ This phenomenon implies that just introducing a timing endogeneity into Lambertini (2002) without a growth rate yields no entry and a perpetual monopoly by the leader.
} 
monotonically changes with an increase in the consumer transport cost parameter. ${ }^{6}$

\section{The Model}

Two firms, $i \in\{1,2\}$, produce homogeneous goods. Consumers are uniformly distributed over the unit segment $[0,1]$ as proposed by Hotelling (1929). ${ }^{7}$ Each consumer at point $x \in$ $[0,1]$ purchases at most one unit of the good and decides from which firm to purchase if he does make a purchase. The consumer at point $x \in[0,1]$ incurs a quadratic transportation cost $c\left(x_{i}-x\right)^{2}$ and pays price $p_{i t}$ at time $t \in[0, \infty)$ when buying a good from firm $i$ located at $x_{i} \in[0,1]$. To summarise, the utility of the consumer at point $x \in[0,1]$ at time $t \in[0, \infty)$ is given by

$$
u_{t}\left(x ; x_{1}, x_{2}, p_{1 t}, p_{2 t}\right)= \begin{cases}\bar{u}-p_{1 t}-c\left(x_{1}-x\right)^{2} & \text { if purchased from firm 1, } \\ \bar{u}-p_{2 t}-c\left(x_{2}-x\right)^{2} & \text { if purchased from firm 2, } \\ 0 & \text { otherwise }\end{cases}
$$

where $\bar{u}$ denotes the gross surplus that a consumer at point $x$ enjoys from purchasing the good, and $c$ is a parameter describing the level of transportation cost or product differentiation. Let us assume that $\bar{u}$ is so large that each consumer prefers to purchase one good over not buying when at least one firm is present in the market. ${ }^{8}$

Assumption $1 \bar{u}>3 c$.

The game proceeds as follows: Each firm $i$ chooses the time of entry $T_{i} \in[0, \infty)$ and location $x_{i} \in[0,1]$ at the same time, and then chooses price $p_{i t}: \Re_{+} \rightarrow \Re_{+}$at each time $t$, which is a function from time $t \in[0, \infty)$ to a real number $[0, \infty)$ and is displayed as $p_{i t}$ for simplicity. In addition, we assume that firm 1 is the leader who just entered at $T_{1}=0$,

\footnotetext{
${ }^{6}$ The transport cost parameter can be interpreted as a parameter that describes the level of product differentiation because the cost parameter corresponds to a consumer's disutility between the consumer's ideal point and the degree of a product's attribute. This interpretation is standard in the literature on spatial economics and marketing.

${ }^{7}$ This setting and the following assumptions are standard in the literature on spatial economics.

${ }^{8}$ In other words, firm 1 , located at $x_{1}=0$, has an incentive to supply a positive amount at location 1 , after maximizing its profit.
} 
whereas firm 2 is the follower who enters at time $T_{2}$, to be subsequently and endogenously determined. In this way, firm 1 decides $x_{1}$ at time $T_{1}=0$ once and subsequently chooses price $p_{1 t}$ at each time $t$. After observing firm 1's actions before firm 2's entry, firm 2 chooses to enter at time $T_{2}$ and location $x_{2}$ and thereafter chooses $p_{2 t}$ at each time $t$. Firm $i$ can choose its location only when it makes its entry in the project, at which time it incurs an entry cost $F_{i}\left(T_{i}\right)$. We also assume (without loss of generality) that $x_{1} \leq 1 / 2$ holds in equilibrium.

Now, let us describe the present value of the firms at time 0 given that firm 2 would enter at point $x_{2}$ at time $t=T_{2}$. Note that firm 1 enters at point $x_{1}$ at time $t=T_{1}=0$. The timing is exogenous ${ }^{9}$ but $x_{1}$ is to be determined endogenously in the following analysis. The total profit of firm 1, the leader, is given by

$$
\begin{aligned}
V_{1}\left(T_{2}, x_{1}, x_{2}, p_{1 t}, p_{2 t}\right)= & \int_{0}^{T_{2}} \int_{0}^{1} p_{1 t}\left(x ; x_{1}\right) e^{-(r-\alpha) t} d x d t \\
& +\int_{T_{2}}^{\infty} \int_{0}^{\bar{x}} p_{1 t}\left(x ; x_{1}, x_{2}, p_{2 t}\right) e^{-(r-\alpha) t} d x d t-F_{1}(0),
\end{aligned}
$$

where $r$ denotes the interest rate, $\alpha$ denotes the growth rate parameter of the market and $\bar{x}$ denotes a point at which the consumer is indifferent between purchasing from firm 1 or 2. From (1), $\bar{x}=\left[p_{2 t}-p_{1 t}+c\left(x_{2}^{2}-x_{1}^{2}\right)\right] /\left[2 c\left(x_{2}-x_{1}\right)\right]$. We assume that $r>\alpha$ to ensure that the follower enters in a finite time. Firm 1 earns monopoly profit flow at $t \in[\tau, \tau+d \tau)$ until firm 2 enters the market, and firm 1 earns duopoly profit flow after firm 2's entry.

The total profit of firm 2, the follower, is given by

$$
V_{2}\left(T_{2}, x_{1}, x_{2}, p_{1 t}, p_{2 t}\right)=\int_{T_{2}}^{\infty} \int_{\bar{x}}^{1} p_{2 t}\left(x ; x_{1}, x_{2}, p_{1 t}\right) e^{-(r-\alpha) t} d x d t-F_{2}\left(T_{2}\right) .
$$

After entry, firm 2 earns duopoly profit flow at $t \in[\tau, \tau+d \tau)$ to which it supplies.

Let us now make the following assumptions for the entry cost function $F_{i}$.

\footnotetext{
${ }^{9} \mathrm{~A}$ similar interpretation is made in Chronopoulos et al. (2014) as a non-preemptive duopoly. In their paper, the roles of the leader and the follower are defined exogenously. Consequently, the future cash flows of the leader are discounted to time $t=0$.
} 
Assumption 2 (i) $F_{i}\left(T_{i}\right)=F_{i} e^{-r T_{i}}$,

(ii) $F_{1}(0)=F_{1}<\int_{0}^{\infty} \int_{0}^{\bar{x}} p_{1 t}\left(x ; x_{1}, x_{2}, p_{2 t}\right) e^{-(r-\alpha) t} d x d t$ for all $x_{2} \in\left[x_{1}, 1\right]$,

(iii) $F_{2}(0)=F_{2}>\int_{0}^{\infty} \int_{\bar{x}}^{1} p_{2 t}\left(x ; x_{1}, x_{2}, p_{1 t}\right) e^{-r t} d x d t$ for all $x_{1} \in\left[0, x_{2}\right]$.

Assumption 2 (ii) suggests that $F_{1}$ is small enough that the leader can earn non-negative total profit at time 0 whenever the follower enters and wherever the follower locates. Similarly, Assumption 2 (iii) suggests that $F_{2}$ is sufficiently large enough to enable the follower to enter sequentially at $T_{2}>0$ in an equilibrium, because we would like to avoid simultaneous entry at time 0 and focus on sequential entry. ${ }^{10}$ This assumption also implies that the leader, but not the follower, has already learned how to enter the market efficiently.

\section{Equilibrium}

In this section, we derive the price, location and timing outcomes in the subgame perfect equilibrium. First, given locations $x_{1}$ and $x_{2}$, we consider the problem of prices at each time $t$ before and after the entry of firm 2. Then, we derive the local profits of the leader and the follower at each time $t$.

The following are the equilibrium prices. Notably, the maximisation of the instantaneous profit flows is equivalent to the maximisation of the total profits. In other words, firm 1 maximises the following equation with respect to $p_{1 t}$ before firm 2 enters $t \in\left[0, T_{2}\right)$ when $T_{2}$ is exogenously given:

$$
\max _{p_{1 t}} \int_{0}^{1} p_{1 t}\left(x ; x_{1}\right) d x
$$

After firm 2 enters $t \in\left[T_{2}, \infty\right)$ when $T_{2}$ is exogenously given, firm 1 maximises the following with respect to $p_{1 t}$ :

$$
\max _{p_{1 t}} \int_{0}^{\bar{x}} p_{1 t}\left(x ; x_{1}, x_{2}, p_{2 t}\right) d x
$$

\footnotetext{
${ }^{10}$ If simultaneous entry occurs, this model reverts to the standard location-price model of d'Aspremont et al. (1979).
} 
and firm 2 maximises the following with respect to $p_{2 t}$ :

$$
\max _{p_{2 t}} \int_{\bar{x}}^{1} p_{2 t}\left(x ; x_{1}, x_{2}, p_{1 t}\right) d x .
$$

Solving these maximisation problems results in the following lemma.

Lemma 1 The prices set by the leader and the follower are

$$
\begin{aligned}
& \tilde{p}_{1 t}= \begin{cases}p_{1}^{M}=\bar{u}-c\left(1-x_{1}\right)^{2} & t \in\left[0, T_{2}\right) \\
p_{1}^{D}=\frac{c}{3}\left(x_{2}-x_{1}\right)\left(2+x_{1}+x_{2}\right) & t \in\left[T_{2}, \infty\right),\end{cases} \\
& \tilde{p}_{2 t}=p_{2}^{D}=\frac{c}{3}\left(x_{2}-x_{1}\right)\left(4-x_{1}-x_{2}\right) \quad t \in\left[T_{2}, \infty\right) \text {. }
\end{aligned}
$$

\section{Proof. See Appendix}

For $t \in\left[0, T_{2}\right)$, the monopoly leader maximises its price under the constraint that all consumers purchase its good. Assuming that $x_{1} \leq 1 / 2$ without loss of generality, the furthest consumer is located at 1 . The consumer turns out to be indifferent between purchasing the good at price $p_{1}^{M}$ and not purchasing the good. $p_{i}^{D}(i=1,2)$ is derived using the standard calculation in the context of spatial competition (e.g. d'Aspremont et al., 1979).

The instantaneous profit flows of the two firms are

$$
\begin{aligned}
& \pi_{1 t}\left(x_{1}, x_{2}\right)= \begin{cases}\pi_{1}^{M}\left(x_{1}\right)=\int_{0}^{1} p_{1}^{M} d x=\bar{u}-c\left(1-x_{1}\right)^{2} & t \in\left[0, T_{2}\right) \\
\pi_{1}^{D}\left(x_{1}, x_{2}\right)=\int_{0}^{\bar{x}} p_{1}^{D} d x=p_{1}^{D} \bar{x}=\frac{c}{18}\left(x_{2}-x_{1}\right)\left(2+x_{1}+x_{2}\right)^{2} & t \in\left[T_{2}, \infty\right),\end{cases} \\
& \pi_{2 t}\left(x_{1}, x_{2}\right)= \begin{cases}0 & t \in\left[0, T_{2}\right) \\
\pi_{2}^{D}\left(x_{1}, x_{2}\right)=\int_{\bar{x}}^{1} p_{2}^{D} d x=p_{2}^{D}(1-\bar{x})=\frac{c}{18}\left(x_{2}-x_{1}\right)\left(4-x_{1}-x_{2}\right)^{2} & t \in\left[T_{2}, \infty\right) .\end{cases}
\end{aligned}
$$

Note that $\bar{x}=\left(2+x_{1}+x_{2}\right) / 6$. Substituting the outcomes of equations (7) to (10) into equations (2) and (3), the total profits of the leader and the follower are derived as

$$
\begin{aligned}
& V_{1}\left(T_{2}, x_{1}, x_{2}, p_{1 t}, p_{2 t}\right)=\int_{0}^{T_{2}} \pi_{1}^{M}\left(x_{1}\right) e^{-(r-\alpha) t} d t+\int_{T_{2}}^{\infty} \pi_{1}^{D}\left(x_{1}, x_{2}\right) e^{-(r-\alpha) t} d t-F_{1} \\
& V_{2}\left(T_{2}, x_{1}, x_{2}, p_{1 t}, p_{2 t}\right)=\int_{T_{2}}^{\infty} \pi_{2}^{D}\left(x_{1}, x_{2}\right) e^{-(r-\alpha) t} d t-F_{2}\left(T_{2}\right) .
\end{aligned}
$$




\subsection{Follower}

We consider the problem of the follower regarding when it enters and where it locates in the market. With regards to the location, we have the following:

Lemma 2 The follower always locates at $x_{2}=1$.

Proof. From equation (12), $x_{2}$ only depends on $\pi_{2}^{D}$. From equation (10), we have

$$
\frac{\partial \pi_{2}^{D}\left(x_{1}, x_{2}\right)}{\partial x_{2}}=\frac{c}{18}\left(4+x_{1}-3 x_{2}\right)\left(4-x_{1}-x_{2}\right)
$$

which is positive for $x_{1}$ and $x_{2} \in[0,1]$. Thus, the optimal location for firm 2 is at 1 , regardless of the other parameters.

From Lemma 2, we show that the follower always locates as far away from the location of the leader as possible when entering the market. This result replicates that of Lambertini (2002) and seems to be robust to the endogeneity of the follower's entry timing. Substituting the equilibrium profits and the location of the follower into the total profit functions $V_{1}$ and $V_{2}$, we have

$$
\begin{aligned}
& V_{1}\left(T_{2}, x_{1}, 1, \tilde{p}_{1 t}, \tilde{p}_{2 t}\right)=\frac{1-e^{-(r-\alpha) T_{2}}}{r-\alpha}\left[\bar{u}-c\left(1-x_{1}\right)^{2}\right]+\frac{e^{-(r-\alpha) T_{2}}}{r-\alpha} \frac{c}{18}\left(1-x_{1}\right)\left(3+x_{1}\right)^{2}-F_{1}, \\
& V_{2}\left(T_{2}, x_{1}, 1, \tilde{p}_{1 t}, \tilde{p}_{2 t}\right)=\frac{e^{-(r-\alpha) T_{2}}}{r-\alpha} \frac{c}{18}\left(1-x_{1}\right)\left(3-x_{1}\right)^{2}-F_{2} e^{-r T_{2}}
\end{aligned}
$$

We note that if $F_{1} \leq F_{2}$, the leader's value is always greater than the follower's value in our setting. That is, since $\pi_{1}^{D} \geq \pi_{2}^{D}$, we have

$$
V_{1}-V_{2} \geq \frac{\pi_{1}^{M}}{r-\alpha}\left(1-e^{-(r-\alpha) T_{2}}\right)-F_{1}\left(1-e^{-r T_{2}}\right)>\left(\frac{\pi_{1}^{M}}{r-\alpha}-F_{1}\right)\left(1-e^{-(r-\alpha) T_{2}}\right)>0 .
$$

We use Assumption 2 (ii) from the end of the last section to ensure $F_{1}<\pi_{1}^{M} /(r-\alpha)$, the present value of profits when the leader sustains monopoly profit forever. 
Thus, the rest of the follower's decision problem is only its endogenously determined entry timing, which is not present in Lambertini (2002). We have the following proposition.

Proposition 1 The entry timing of the follower is

$$
\tilde{T}_{2}\left(x_{1}\right)=\frac{1}{\alpha} \log \left[\frac{r F_{2}}{\pi_{2}^{D}\left(x_{1}, 1\right)}\right]=\frac{1}{\alpha} \log \left[\frac{18 r F_{2}}{c\left(1-x_{1}\right)\left(3-x_{1}\right)^{2}}\right] .
$$

Proof. Differentiating $V_{2}\left(T_{2}, x_{1}, 1, \tilde{p}_{1 t}, \tilde{p}_{2 t}\right)$ with respect to $T_{2}$ yields

$$
\frac{\partial V_{2}\left(T_{2}, x_{1}, 1, \tilde{p}_{1 t}, \tilde{p}_{2 t}\right)}{\partial T_{2}}=-e^{-(r-\alpha) T_{2}} \pi_{2}^{D}\left(x_{1}, 1\right)+r F_{2} e^{-r T_{2}}=0 .
$$

Solving this equation with respect to $T_{2}$ gives $\tilde{T}_{2}\left(x_{1}\right)$ as a solution. Note that $\tilde{T}_{2}$ is positive from Assumption 2 (iii). Examining the second-order derivative yields the following

$$
\frac{\partial^{2} V_{2}}{\partial T_{2}^{2}}=(r-\alpha) e^{-(r-\alpha) T_{2}} \pi_{2}^{D}\left(x_{1}, 1\right)-r^{2} F_{2} e^{-r T_{2}} .
$$

Substituting $\tilde{T}_{2}\left(x_{1}\right)$ into the second-order derivative shows that $\partial^{2} V_{2} /\left.\partial T_{2}^{2}\right|_{T_{2}=\tilde{T}_{2}\left(x_{1}\right)}$ is negative. Because the first-order condition is uniquely satisfied and the second-order derivative is negative at this point, the unique, positive and interior solution exists. Thus, we have the desired result.

The following corollaries show the change in the follower's entry timing as the leader's location $x_{1}$ and the exogenous parameters change.

Corollary 1 If $x_{1}$ is increased, the optimal timing for the follower to enter is delayed.

$x_{1}$ affects the entry timing of the follower as follows. Only the denominator within the fraction inside the log in equation (17) is composed of the locations chosen by the firms. Thus, we focus on this part of the equation, namely, $\pi_{2}^{D}\left(x_{1}, 1\right)$. When firm 1 locates away from firm 2 ( or $x_{1}$ is decreased), firm 2 can deliver the product in a broader region $(\bar{x}$ decreases), and firm 2 can earn a higher profit ( $\pi_{2}^{D}$ increases). Therefore, firm 2 enters earlier (later) if firm 1 locates away from (closer to) firm 2. 
Corollary 2 If $F_{2}$ or $r$ is increased, or as $c$ or $\alpha$ is decreased, the optimal timing for the follower to enter is delayed.

As is seen in the next subsection, $x_{1}$ depends on the previous parameters. Therefore, a change in these parameters affects the entry timing of the follower through a direct effect (Corollary 2) and an indirect effect (Corollary 1).

\subsection{Leader}

Finally, we consider the problem of the leader. Substituting the outcomes of the follower, Lemma 2 and Proposition 1 into the total profit function of the leader, the maximisation problem of the leader is given by

$$
\begin{aligned}
\max _{x_{1} \in[0,1 / 2]} & V_{1}\left(\tilde{T}_{2}\left(x_{1}\right), x_{1}, 1, \tilde{p}_{1 t}, \tilde{p}_{2 t}\right) \\
& =\frac{1-e^{-(r-\alpha) \tilde{T}_{2}\left(x_{1}\right)}}{r-\alpha}\left[\bar{u}-c\left(1-x_{1}\right)^{2}\right]+\frac{e^{-(r-\alpha) \tilde{T}_{2}\left(x_{1}\right)}}{r-\alpha} \frac{c}{18}\left(1-x_{1}\right)\left(2+x_{1}+1\right)^{2}-F_{1} .
\end{aligned}
$$

Because the derivation of the equilibrium is complicated, we investigate the impact on the value of the leader when the location of the leader changes infinitesimally, before deriving

the equilibrium outcome presented in Proposition 2. Differentiating $V_{1}\left(\tilde{T}_{2}\left(x_{1}\right), x_{1}, 1, \tilde{p}_{1 t}, \tilde{p}_{2 t}\right)$ with respect to $x_{1}$ yields

$$
\begin{gathered}
\frac{\partial V_{1}\left(\tilde{T}_{2}\left(x_{1}\right), x_{1}, 1, \tilde{p}_{1 t}, \tilde{p}_{2 t}\right)}{\partial x_{1}}=e^{-(r-\alpha) \tilde{T}_{2}\left(x_{1}\right)} \frac{d \tilde{T}_{2}\left(x_{1}\right)}{d x_{1}}\left(\pi_{1}^{M}\left(x_{1}\right)-\pi_{1}^{D}\left(x_{1}, 1\right)\right) \\
+\frac{1-\exp \left(-(r-\alpha) \tilde{T}_{2}\left(x_{1}\right)\right)}{r-\alpha} \frac{d \pi_{1}^{M}\left(x_{1}\right)}{d x_{1}} \\
+\frac{\exp \left(-(r-\alpha) \tilde{T}_{2}\left(x_{1}\right)\right)}{r-\alpha} \frac{d \pi_{1}^{D}\left(x_{1}, 1\right)}{d x_{1}} .
\end{gathered}
$$

The sign of equation (21) is the key and determines the location of the leader: whether it is located at the centre (1/2), edge (0), or an interior point (strictly between 0 and $1 / 2$ ). The first term of (21) represents the gain from the delay of entry by firm 2 that is caused by an increase in $x_{1}$, allowing firm 1 to maintain its monopoly profit before the duopoly 
regime begins. This term is not present in Lambertini (2002) and captures the essence behind our results. Therefore, if this term turns out to be large, our result may become significantly different from that of Lambertini (2002). The second term of (21) signifies the increase in the monopoly profit attributable to moving closer to the centre. The third term of (21) shows how the duopoly profit decreases as firm 1 moves closer to firm 2, thus intensifying competition. ${ }^{11}$ The first two terms are positive and the last term is negative. Thus, if the effect of the last term is relatively small, the optimal location of firm 1 is $1 / 2$.

We now investigate the effect of each parameter on the equilibrium location of firm 1 , which we denote as $x_{1}^{E}$. First, consider the effects of parameters $\alpha$ and $r$ on $x_{1}^{E}$. As $\alpha$ approaches $r$, the weight on the periods after which firm 2 enters the market gets larger. Therefore, firm 1 needs to take into account profitability under the duopoly situation. The significance of the last term dominates that of the other two terms, which implies that the optimal location of firm 1 is $x_{1}=0$. In contrast, if $\alpha$ becomes smaller, the converse holds. Thus, the effect of parameter $r$ is essentially the opposite of that of $\alpha$.

Parameter $\bar{u}$ appears only in the first term, which is increasing in $\bar{u}$. As the location of firm 1 becomes closer to the centre, the leader obtains the monopoly profit for a longer time because the follower's timing of entry is delayed from Corollary 1. An increase in $\bar{u}$ yields a stronger incentive to obtain this profit. Thus, as $\bar{u}$ increases, the leader is more likely to locate at $x_{1}^{E}=1 / 2$.

Regarding $F_{2}$ and $c$, they affect equation (21) through $\tilde{T}_{2}$ in that an increase in $F_{2}$ or a decrease in $c$ increases $\tilde{T}_{2}$. Thus, the effects of these two parameters counter each other. Note that the term $d \tilde{T}_{2}\left(x_{1}\right) / d x_{1}$ is independent of $c$ or $F_{2}$. From Corollary 2, increasing $F_{2}$ delays the entry of the follower (increasing $\tilde{T}_{2}$ ), and $F_{2}$ does not affect equation (21) in other ways. The discounted present value of the increase in profit from a longer monopoly regime attributable to firm 1 locating closer to the centre is decreased. Thus, the first term is decreasing in $F_{2}$. The second term is increasing in $F_{2}$ because the increase in the

\footnotetext{
${ }^{11}$ Although $T_{2}$ is not endogenised, the second and third terms describe how Lambertini (2002) determined the leader's location.
} 
monopoly profit for firm 1 from its moving closer to the centre is sustained longer due to the entry delay. Similarly, the third term is also increasing in $F_{2}$, as the decrease in the duopoly profit for firm 1 from its moving closer to the centre is devalued from the entry delay. These three effects are complicated, and none of them are analytically dominant. We numerically examine this issue in the next section.

The effect of parameter $c$ counters that of $F_{2}$ with respect to how they affect $\tilde{T}_{2}$. In addition, $c$ enters in all the profit levels. Namely, $c$ has two contrasting effects on the profit of firm 1. First, the monopoly profit of firm 1 is decreasing in $c$. When the firm supplies to all consumers, it needs to compensate consumers for transport costs by lowering its monopoly price. The compensation is higher as the consumer transport cost parameter increases. Second, the parameter $c$ is positively related to the duopoly profit of firm 1 as in the standard Hotelling model with price competition. The effect of the first term is lower as the parameter $c$ increases, whereas those of the second and third terms are higher. Therefore, the relative importance of the three terms influences the effect of $c$ on the location choice of firm 1. Unlike the previous argument, this effect depends on the parameters $\alpha$ and $r$. We apply the previous argument regarding $\alpha$ to the effect of parameter $c$ on the optimal location of firm 1. If $\alpha$ approaches $r$, the significance of the last term dominates that of the second term, which implies that the optimal location of firm 1 is more likely to become $x_{1}^{E}=0$ as $c$ increases. In contrast, if $\alpha$ becomes smaller, the significance of the second term dominates the last, which implies that the optimal location of firm 1 is more likely to become $x_{1}^{E}=1 / 2$ as $c$ increases. If $r$ is sufficiently large, for example $r>1+\alpha$, the optimal location of firm 1 is more likely to become $x_{1}^{E}=0$ as $c$ increases because the first term is relatively large.

Solving (20), we have the following proposition.

Proposition 2 (a) If (21) is positive for any $x_{1} \in[0,1 / 2]$, the outcome of the subgame 
perfect equilibrium is

$$
\begin{aligned}
& T_{2}^{*}=\frac{1}{\alpha} \log \left[\frac{144 r F_{2}}{25 c}\right], x_{1}^{*}=\frac{1}{2}, x_{2}^{*}=1, \bar{x}^{*}=\frac{7}{12}, \\
& p_{1 t}^{*}=\left\{\begin{array}{ll}
\tilde{p}_{1}^{M}\left(x_{1}^{*}\right)=\bar{u}-\frac{c}{4} & t \in\left[0, T_{2}^{*}\right) \\
p_{1}^{D}\left(x_{1}^{*}, x_{2}^{*}\right)=\frac{7 c}{12} & t \in\left[T_{2}^{*}, \infty\right),
\end{array} p_{2 t}^{*}=p_{2}^{D}\left(x_{1}^{*}, x_{2}^{*}\right)=\frac{5 c}{12} \quad t \in\left[T_{2}^{*}, \infty\right) .\right.
\end{aligned}
$$

(b) If (21) is negative for any $x_{1} \in[0,1 / 2]$, the outcome of the subgame perfect equilibrium is

$$
\begin{aligned}
T_{2}^{* *} & =\frac{1}{\alpha} \log \left[\frac{2 r F_{2}}{c}\right], x_{1}^{* *}=0, x_{2}^{* *}=1, \bar{x}^{* *}=\frac{1}{2}, \\
p_{1 t}^{* *} & =\left\{\begin{array}{ll}
p_{1}^{M}\left(x_{1}^{* *}\right)=\bar{u}-c & t \in\left[0, T_{2}^{* *}\right) \\
p_{1}^{D}\left(x_{1}^{* *}, x_{2}^{* *}\right)=c & t \in\left[T_{2}^{* *}, \infty\right),
\end{array} p_{2 t}^{* *}=p_{2}^{D}\left(x_{1}^{* *}, x_{2}^{* *}\right)=c \quad t \in\left[T_{2}^{* *}, \infty\right) .\right.
\end{aligned}
$$

(c) If the equilibrium location of firm 1, $x_{1}^{* * *}$, is strictly between 0 and 1/2, then the outcome of subgame perfect equilibrium is

$$
\begin{aligned}
& T_{2}^{* * *}=\frac{1}{\alpha} \log \left[\frac{18 r F_{2}}{c\left(1-x_{1}^{* * *}\right)\left(3-x_{1}^{* * *}\right)^{2}}\right], x_{2}^{* * *}=1, \bar{x}^{* * *}=\frac{3+x_{1}^{* * *}}{6}, \\
& p_{1 t}^{* * *}= \begin{cases}p_{1}^{M}\left(x_{1}^{* * *}\right)=\bar{u}-c\left(1-x_{1}^{* * *}\right)^{2} & t \in\left[0, T_{2}^{* * *}\right) \\
p_{1}^{D}\left(x_{1}^{* * *}, x_{2}^{* * *}\right)=\frac{c}{3}\left(1-x_{1}^{* * *}\right)\left(3+x_{1}^{* * *}\right) & t \in\left[T_{2}^{* * *}, \infty\right),\end{cases} \\
& p_{2 t}^{* * *}=p_{2}^{D}\left(x_{1}^{* * *}, x_{2}^{* * *}\right)=\frac{c}{3}\left(1-x_{1}^{* * *}\right)\left(3-x_{1}^{* * *}\right) \quad t \in\left[T_{2}^{* * *}, \infty\right) .
\end{aligned}
$$

Proposition 2 shows that three types of equilibrium location for the leader can emerge. Similar to Lambertini (2002), we show that firm 1 can locate at 0, 0.5 or an interior location depending on the parameter values. However, a case exists in which the introduction of endogenous entry timing leads to different equilibrium location outcomes compared with Lambertini (2002) using the same parameter values. To grasp the intuition behind this result, we proceed with a numerical analysis in the next section.

\section{Numerical Analysis}

In this section, we investigate in detail the underlying properties of our model using numerical analysis. First, we investigate the effects of the key parameters, $\alpha, c$ and $F_{2}$, 
on firm 1's equilibrium location, which we denote as $x_{1}^{E} \in\left\{x_{1}^{*}, x_{1}^{* *}, x_{1}^{* * *}\right\}$. Then, we show the importance of the endogeneity of $T_{2}$, namely the first term of equation (21). Finally, we examine the effects of the parameters on the total values of the firms in equilibrium.

\subsection{The leader's location}

This subsection examines the effects of parameters $\left(\alpha, c, F_{2}\right)$ on the equilibrium location of the leader.

First, let us consider the effect of $\alpha$ on $x_{1}^{E}$. Let us set the parameters as $r=0.1, \bar{u}=4$ and $c=1$. We use three tables to illustrate the relationship between $\alpha$ and the equilibrium location for the leader. $F_{2}=20,50$ and 100 for Tables 1 to 3, respectively. The values of $\alpha$ are incremented by one ten-thousandth. As explained in the previous section, we pointed out that the location $x_{1}^{E}$ moves from $1 / 2$ to 0 as $\alpha$ approaches $r$. We confirm this result from all three tables.

Additionally, we investigate the effects of $F_{2}$ on $x_{1}^{E}$ when $\alpha$ approaches $r$. $\bar{\alpha}$ denotes the lowest value for which the equilibrium $x_{1}^{E}$ is 0 , and $\underline{\alpha}$ denotes the highest value for which the equilibrium $x_{1}^{E}$ is $1 / 2$. Tables 1 to 3 imply that both $\bar{\alpha}$ and $\underline{\alpha}$ are increasing in $F_{2}$. This implication is consistent with the intuition offered in the previous section. In addition, the range of the interior location, $\bar{\alpha}-\underline{\alpha}$, is increasing in $F_{2}$. Therefore, as $F_{2}$ increases, the equilibrium location is likely to become interior or $1 / 2$ and not 0 .

\begin{tabular}{|c||c|c|c|c|c|c|c|c|c|}
\hline$\alpha$ & $\cdots$ & 0.0961 & 0.0962 & $\cdots$ & 0.0969 & $\cdots$ & 0.0976 & 0.0977 & $\cdots$ \\
\hline$x_{1}^{E}$ & 0.5 & 0.5 & 0.432 & $\cdots$ & 0.171 & $\cdots$ & 0.014 & 0 & 0 \\
\hline
\end{tabular}

Table 1: Location of firm $1, x_{1}$, when $r=0.1, \bar{u}=4, F_{2}=20$, and $c=1$. We have $\underline{\alpha}=0.0961, \bar{\alpha}=0.0977$.

Second, we investigate the effect of $c$ on the equilibrium location of the leader. Figure 1 illustrates the equilibrium location of firm 1 for different values of $c$ when $F_{2}=20$ and $F_{2}=13$. Consider the case in which an interior solution can exist. An interior solution exists when $\alpha$ approaches $r$. We consider the same values as in the previous numerical 


\begin{tabular}{|c||c|c|c|c|c|c|c|c|c|}
\hline$\alpha$ & $\cdots$ & 0.0964 & 0.0965 & $\cdots$ & 0.0973 & $\cdots$ & 0.0981 & 0.0982 & $\cdots$ \\
\hline$x_{1}^{E}$ & 0.5 & 0.5 & 0.453 & $\cdots$ & 0.192 & $\cdots$ & 0.011 & 0 & 0 \\
\hline
\end{tabular}

Table 2: Location of firm $1, x_{1}$, when $r=0.1, \bar{u}=4, F_{2}=50$, and $c=1$. We have $\underline{\alpha}=0.0964, \bar{\alpha}=0.0982$.

\begin{tabular}{|c||c|c|c|c|c|c|c|c|c|}
\hline$\alpha$ & $\cdots$ & 0.0966 & 0.0967 & $\cdots$ & 0.975 & $\cdots$ & 0.0983 & 0.0984 & $\cdots$ \\
\hline$x_{1}^{E}$ & 0.5 & 0.5 & 0.46 & $\cdots$ & 0.212 & $\cdots$ & 0.024 & 0 & 0 \\
\hline
\end{tabular}

Table 3: Location of firm $1, x_{1}^{E}$, when $r=0.1, \bar{u}=4, F_{2}=100$, and $c=1$. We have $\underline{\alpha}=0.0966, \bar{\alpha}=0.0984$.

analysis; $r=0.1, \alpha=0.096$ and $\bar{u}=4$. As $\bar{u}>3 c, c \in(0,4 / 3)$. When $F_{2}=20$, the value of $x_{1}^{E}$ declines from $1 / 2$ to approximately $1 / 10$ as $c$ increases. In this case, firm 1 never locates at point 0 . When $F_{2}=13, x_{1}^{E}$ moves from $1 / 2$ to 0 as $c$ increases. Figure 1 demonstrates the following concept. If the interior solution exists in the equilibrium, $x_{1}^{E}$ is decreasing in $c$. As $F_{2}$ increases, $x_{1}^{E}$ is more likely to take a higher value for a given $c$.

Third, we show the effects of both $c$ and $F_{2}$ on the equilibrium location of the leader. Tables 4 and 5 illustrate the equilibrium location of firm 1 for different values of $c$ and $F_{2}$ when $r=0.1, \bar{u}=4$ and $\alpha=0.098$ for Table 4 and $\alpha=0.097$ for Table 5 . For these values of $r$ and $\bar{u}$, these values of $\alpha$ allow interior solutions of $x_{1}$. Thus, for $\alpha$ very close to $r$, at approximately 0.099 , we primarily have $x_{1}=0$; however, for most values of $\alpha$, we tend to have the other corner solution at $x_{1}=1 / 2$.

By examining the interior solutions, we can better understand the effects of $c$ and $F_{2}$. Values of $c$ increase by 0.1 up to 1.3 , as $\bar{u}>3 c, c \in(0,4 / 3) . F_{2}$ is increased from 10 to 200 in increments of 10. Both tables show two trends. As $c$ increases, if an interior solution exists in the equilibrium, $x_{1}^{E}$ decreases. As $F_{2}$ increases, $x_{1}^{E}$ increases if the interior solution exists in the equilibrium. As noted in the previous section, the effects of these two parameters tend to counter each other. 


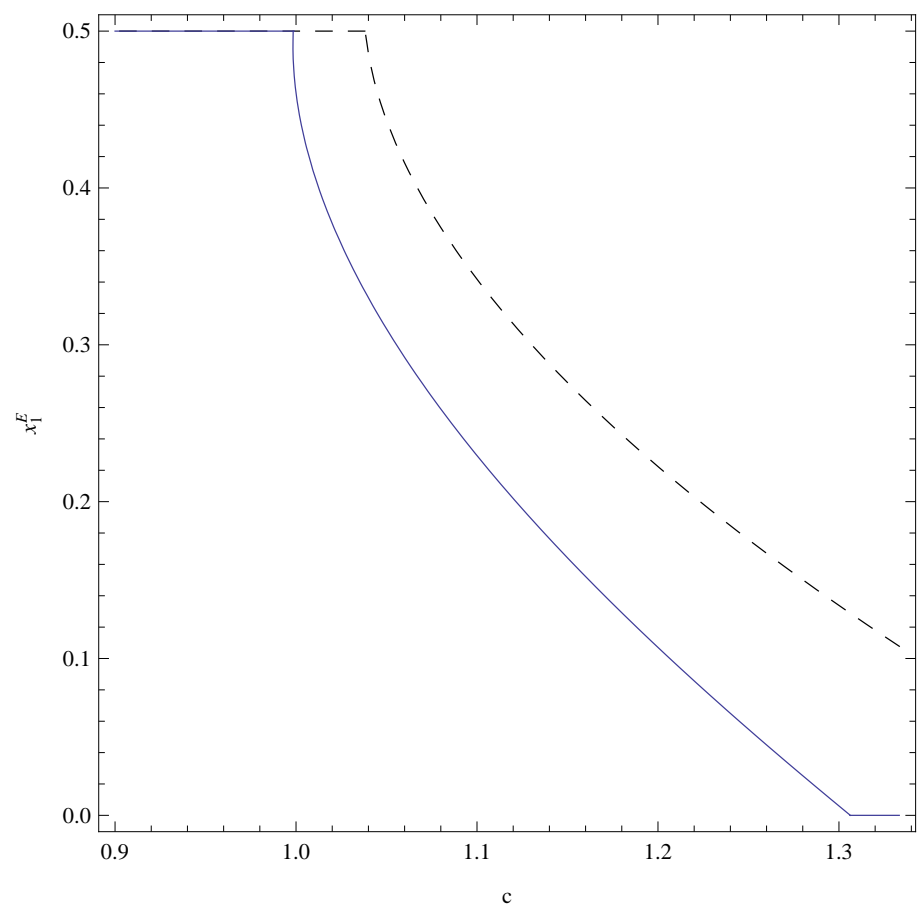

Figure 1: Plotted values of optimal location for firm 1 with respect to $c \in(9 / 10,4 / 3)$ when $F_{2}=20$ (dotted) and $F_{2}=13$ (solid).

\subsection{On the importance of endogenous timing by the follower}

One of the main points of this paper in contrast to previous papers, including Lambertini (2002), is that the follower's entry timing is endogenised and the leader determines its location considering this move. To determine its importance, we examine the relative sizes of the three terms in equation (21). Equation (21) is the first-order derivative of $V_{1}$ with respect to $x_{1}$. The first term represents how the marginal change in the leader's location delays the follower's entry, allowing the leader to prolong its monopoly regime.

Using the parameters and the leader's location $x_{1}$ in Table 2, we derive concrete values of equation (21) in four cases. The common parameter values are $r=0.1, \bar{u}=4, c=1$ and $F_{2}=50$. The result is summarised in Table 6 .

The sum column in Table 6 indicates how firm 1 decides its location. If the value in the column is negative (positive), then firm 1 locates at $0(0.5)$. If it is 0 , an interior 


\begin{tabular}{|c||c|c|c|c|c|c|c|c|c|c|}
\hline$F_{2} / c$ & 0.4 & 0.5 & 0.6 & 0.7 & 0.8 & 0.9 & 1.0 & 1.1 & 1.2 & 1.3 \\
\hline \hline 200 & 0.5 & 0.5 & 0.5 & 0.5 & 0.5 & 0.5 & 0.418 & 0.353 & 0.304 & 0.265 \\
\hline 190 & 0.5 & 0.5 & 0.5 & 0.5 & 0.5 & 0.5 & 0.414 & 0.348 & 0.299 & 0.260 \\
\hline 180 & 0.5 & 0.5 & 0.5 & 0.5 & 0.5 & 0.5 & 0.408 & 0.343 & 0.294 & 0.255 \\
\hline 170 & 0.5 & 0.5 & 0.5 & 0.5 & 0.5 & 0.5 & 0.403 & 0.338 & 0.288 & 0.249 \\
\hline 160 & 0.5 & 0.5 & 0.5 & 0.5 & 0.5 & 0.5 & 0.397 & 0.332 & 0.283 & 0.243 \\
\hline 150 & 0.5 & 0.5 & 0.5 & 0.5 & 0.5 & 0.497 & 0.391 & 0.323 & 0.276 & 0.236 \\
\hline 140 & 0.5 & 0.5 & 0.5 & 0.5 & 0.5 & 0.489 & 0.384 & 0.319 & 0.269 & 0.229 \\
\hline 130 & 0.5 & 0.5 & 0.5 & 0.5 & 0.5 & 0.480 & 0.377 & 0.312 & 0.262 & 0.222 \\
\hline 120 & 0.5 & 0.5 & 0.5 & 0.5 & 0.5 & 0.470 & 0.369 & 0.303 & 0.254 & 0.213 \\
\hline 110 & 0.5 & 0.5 & 0.5 & 0.5 & 0.5 & 0.460 & 0.360 & 0.295 & 0.245 & 0.204 \\
\hline 100 & 0.5 & 0.5 & 0.5 & 0.5 & 0.5 & 0.448 & 0.350 & 0.285 & 0.234 & 0.193 \\
\hline 90 & 0.5 & 0.5 & 0.5 & 0.5 & 0.5 & 0.436 & 0.339 & 0.273 & 0.223 & 0.181 \\
\hline 80 & 0.5 & 0.5 & 0.5 & 0.5 & 0.5 & 0.422 & 0.326 & 0.261 & 0.210 & 0.167 \\
\hline 70 & 0.5 & 0.5 & 0.5 & 0.5 & 0.5 & 0.406 & 0.311 & 0.246 & 0.194 & 0.151 \\
\hline 60 & 0.5 & 0.5 & 0.5 & 0.5 & 0.5 & 0.387 & 0.294 & 0.228 & 0.175 & 0.132 \\
\hline 50 & 0.5 & 0.5 & 0.5 & 0.5 & 0.5 & 0.365 & 0.272 & 0.205 & 0.152 & 0.107 \\
\hline 40 & 0.5 & 0.5 & 0.5 & 0.5 & 0.5 & 0.334 & 0.244 & 0.176 & 0.122 & 0.075 \\
\hline 30 & 0.5 & 0.5 & 0.5 & 0.5 & 0.467 & 0.298 & 0.206 & 0.136 & 0.079 & 0.030 \\
\hline 20 & 0.5 & 0.5 & 0.5 & 0.5 & 0.400 & 0.240 & 0.15 & 0.072 & 0.011 & 0 \\
\hline 10 & 0.5 & 0.5 & 0.5 & 0.5 & 0.266 & 0.123 & 0.191 & 0 & 0 & 0 \\
\hline
\end{tabular}

Table 4: Location of firm $1, x_{1}^{E}$, depending on the values of $F_{2}$ and $c$, when $r=0.1, \alpha=$ $0.097, \bar{u}=4$.

location between 0 and 0.5 may arise.

The last three cases are interesting. If the first term is not present, the sum will be negative, and firm 1 has an incentive to move closer to 0 . Therefore, compared with Lambertini (2002), firm 1 is more likely to locate closer to the centre, forcing firm 2 to delay its entry. This effect of delaying entry does not exist in Lambertini (2002).

\subsection{On total values of the firms}

Finally, from the firms' point of view, their total values are more or at least as important as their entry timing and locations. We examine how the parameters $\bar{u}$ and $c$, commonly used in the Hotelling settings, affect firm values. The result for $\bar{u}$ is straightforward, but 


\begin{tabular}{|c||c|c|c|c|c|c|c|c|c|c|}
\hline$F_{2} / c$ & 0.4 & 0.5 & 0.6 & 0.7 & 0.8 & 0.9 & 1.0 & 1.1 & 1.2 & 1.3 \\
\hline \hline 200 & 0.5 & 0.5 & 0.420 & 0.305 & 0.235 & 0.184 & 0.144 & 0.112 & 0.084 & 0.061 \\
\hline 190 & 0.5 & 0.5 & 0.417 & 0.302 & 0.231 & 0.180 & 0.140 & 0.108 & 0.080 & 0.057 \\
\hline 180 & 0.5 & 0.5 & 0.413 & 0.298 & 0.228 & 0.177 & 0.136 & 0.104 & 0.076 & 0.053 \\
\hline 170 & 0.5 & 0.5 & 0.409 & 0.294 & 0.224 & 0.173 & 0.132 & 0.099 & 0.072 & 0.048 \\
\hline 160 & 0.5 & 0.5 & 0.404 & 0.290 & 0.220 & 0.168 & 0.128 & 0.095 & 0.067 & 0.043 \\
\hline 150 & 0.5 & 0.5 & 0.400 & 0.286 & 0.215 & 0.164 & 0.123 & 0.090 & 0.062 & 0.038 \\
\hline 140 & 0.5 & 0.5 & 0.395 & 0.281 & 0.210 & 0.159 & 0.118 & 0.085 & 0.056 & 0.032 \\
\hline 130 & 0.5 & 0.5 & 0.389 & 0.276 & 0.205 & 0.153 & 0.112 & 0.079 & 0.050 & 0.026 \\
\hline 120 & 0.5 & 0.5 & 0.383 & 0.271 & 0.200 & 0.147 & 0.106 & 0.072 & 0.044 & 0.019 \\
\hline 110 & 0.5 & 0.5 & 0.377 & 0.265 & 0.193 & 0.141 & 0.099 & 0.065 & 0.037 & 0.012 \\
\hline 100 & 0.5 & 0.5 & 0.370 & 0.258 & 0.186 & 0.133 & 0.092 & 0.058 & 0.029 & 0.004 \\
\hline 90 & 0.5 & 0.5 & 0.362 & 0.250 & 0.178 & 0.125 & 0.083 & 0.049 & 0.019 & 0 \\
\hline 80 & 0.5 & 0.5 & 0.353 & 0.242 & 0.170 & 0.116 & 0.074 & 0.039 & 0.009 & 0 \\
\hline 70 & 0.5 & 0.5 & 0.341 & 0.232 & 0.159 & 0.105 & 0.062 & 0.027 & 0 & 0 \\
\hline 60 & 0.5 & 0.5 & 0.331 & 0.220 & 0.147 & 0.092 & 0.049 & 0.013 & 0 & 0 \\
\hline 50 & 0.5 & 0.5 & 0.317 & 0.206 & 0.132 & 0.077 & 0.033 & 0 & 0 & 0 \\
\hline 40 & 0.5 & 0.5 & 0.299 & 0.188 & 0.113 & 0.057 & 0.012 & 0 & 0 & 0 \\
\hline 30 & 0.5 & 0.5 & 0.275 & 0.163 & 0.087 & 0.030 & 0 & 0 & 0 & 0 \\
\hline 20 & 0.5 & 0.5 & 0.239 & 0.127 & 0.048 & 0 & 0 & 0 & 0 & 0 \\
\hline 10 & 0.5 & 0.399 & 0.173 & 0.056 & 0 & 0 & 0 & 0 & 0 & 0 \\
\hline
\end{tabular}

Table 5: Location of firm $1, x_{1}^{E}$, depending on the values of $F_{2}$ and $c$ when $r=0.1, \alpha=$ $0.098, \bar{u}=4$.

\begin{tabular}{|c||c|c|c|c||c|}
\hline & $x_{1}^{E}$ & First term & Second term & Third term & Sum \\
\hline$\alpha=0.0985$ & 0 & 40.844 & 45.943 & -107.283 & -20.496 \\
\hline$\alpha=0.0973$ & 0.192 & 53.826 & 42.398 & -96.224 & 0 \\
\hline$\alpha=0.0965$ & 0.453 & 80.420 & 34.584 & -115.004 & 0 \\
\hline$\alpha=0.092$ & 0.5 & 77.479 & 31.673 & -45.367 & 63.785 \\
\hline
\end{tabular}

Table 6: Values of the three terms in equation (21) when $r=0.1, \bar{u}=4, F_{2}=50$, and $c=1$.

that for $c$ is more complicated.

An increase in $\bar{u}$ improves the leader's value and possibly worsens the follower's value. $\bar{u}$ only appears in the monopoly profit phase of the leader. As it increases, firm 1 has 
more incentive to move closer to the centre, giving firm 2 an incentive to delay its entry if the leader has not already located at the centre. Because there is only one effect to be considered, the result is simple.

Examining the impact of the change in $c$ on the values of the leader and follower requires greater care. We set the parameters as $r=0.1$ and $\bar{u}=4$. Figure 2 demonstrates that the value of the follower is monotonically increasing in $c$ whereas that of the leader does not move monotonically. In a Hotelling setting, transport cost $c$ can be interpreted as the degree of product differentiation. A larger $c$ indicates a greater degree of product differentiation.

In our model, if $c$ increases, the follower always enjoys a positive effect on its total profit, which is not always true for the leader because the effects of an increase in $c$ are classified into three categories: (i) a decrease in $T_{2}$, (ii) a decrease in $\pi_{1}^{M}$ and (iii) an increase in $\pi_{1}^{D}$. In other words, as product space becomes more differentiated, (i) the follower enters earlier, (ii) the monopoly profit decreases and (iii) the duopoly profits after entry increase. The follower only faces (i) and (iii), which are both positive effects for the follower, yielding that $V_{2}$ is increasing in $c$. However, effects (i) and (ii) are negative for the leader, whereas effect (iii) is positive. As $c$ increases, (ii) and (iii), presented by $\pi_{1}^{M}$ and $\pi_{1}^{D}$ respectively, are linearly changed whereas (i), presented by $\tilde{T}_{2}$, decreases proportionally to $-\log c$. Thus, when $c$ is close to zero, the effect (i) dominates (iii), and $V_{1}$ is decreasing in $c$. When $c$ is relatively large, effect (i) is small, effect (iii) dominates (i) and (ii) and $V_{1}$ is increasing in $c$.

In the usual static Hotelling game, only effect (iii) exists, yielding that greater differentiation on a product space always enlarges the values of duopoly firms. This result is well known in the related literature. However, in our dynamic Hotelling game, greater differentiation in a product space may not benefit both firms, thus indicating the importance of introducing timing into the consideration. 


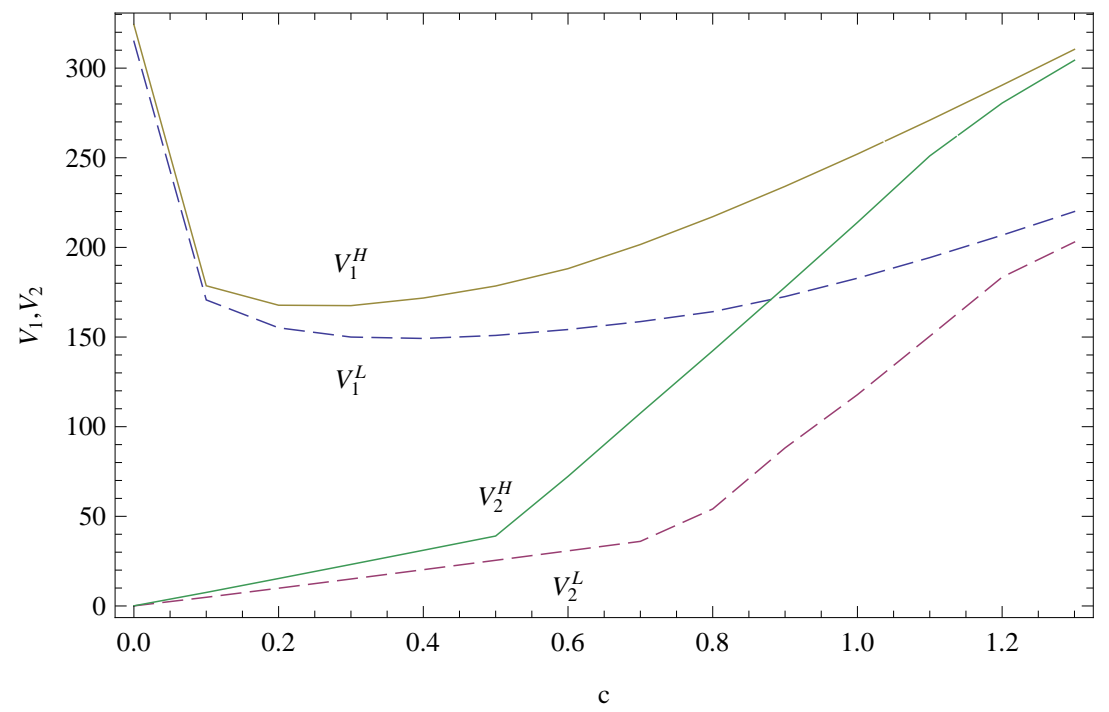

Figure 2: Plotted values of the values of the leader and the follower with respect to $c \in\{0.001,0.1,0.2,0.3, \cdots, 1.3\}$, when $r=0.1, \bar{u}=4 . V_{1}^{H}$ and $V_{2}^{H}$ are the values of $V_{1}$ and $V_{2}$ when $\alpha=0.098$ and $F_{1}=F_{2}=60 . V_{1}^{L}$ and $V_{2}^{L}$ are the values of $V_{1}$ and $V_{2}$ when $\alpha=0.097$ and $F_{1}=F_{2}=20$.

\section{Concluding Remarks}

In this paper, we develop a duopoly model that determines the follower's entry timing, firms' locations and their prices. Examining the timing of investments is important when considering firms' entry strategies. Hence, we extend Lambertini (2002), which in turn extends the location-price competition model (d'Aspremont et al., 1979) by using a continuous time model in which firms earn profits in each instance and the follower's entry timing is given exogenously. Our model endogenises the follower's entry timing. In doing so, we introduce parameters such as investment costs and a market growth rate to make the model and the outcome more realistic.

We find that these changes create a strategic interaction between the leader's location and the follower's entry timing. As a result, the leader has greater incentive to locate closer to the centre to delay the follower's entry. We also find that the follower always locates as far away as possible from the leader, which is a robust result also seen in Lam- 
bertini (2002). Numerical analyses are also presented that investigate how the exogenous parameters affect the leader's location and the firms' values. In particular, the profit of the leader changes non-monotonically with an increase in the transport cost parameter.

A potential future study should address the last result, for which the relationship between the firm values and the transport cost parameter $c$ is nontrivial. $c$ can be interpreted as a parameter that describes the degree of product differentiation. Therefore, $c$ is important for firms and their marketing strategies, necessitating its careful estimation. Another issue that needs to be addressed is making the entry timing fully endogenous. In this paper, we allow the follower to enter endogenously; however, the game starts when the leader enters at $T=0$, thus exogenously. This setting is intentional in order to compare our result to Lambertini (2002). However, we may allow the leader to endogenously enter and, more interestingly, allow a preemption by the firms to enable full analysis of the endogenous entry timing model, as indicated by Fudenberg and Tirole (1985).

\section{Appendix}

\section{Proof of Lemma 1:}

The duopoly result is a straightforward maximisation of profit by the two firms, as previously stated. See, for example, d'Aspremont et al., (1979).

As for a monopoly, firm 1 is located at or to the left of $1 / 2$, which we denote as $x_{1}$. Firm 1 sets up its price so that the consumer at 1 or at a point to the left of 1 is indifferent between purchasing and not purchasing. That is, the consumer at 1 has no surplus, as otherwise firm 1 has an incentive to increase its price without losing any of its consumers. Let this indifferent consumer be denoted $\hat{x}$.

Firm 1's profit is given by

$$
\begin{array}{r}
\pi_{1}=p_{1} \hat{x}, \\
\text { s.t. } \quad \bar{u}-p_{1}-c\left(\hat{x}-x_{1}\right)^{2}=0 .
\end{array}
$$


Substituting $p_{1}$ of the constraint into the profit and maximising with respect to $\hat{x}$ gives

$$
\frac{\left.\partial \pi_{1}\right|_{p_{1}}}{\partial \hat{x}}=\bar{u}-c\left(3 \hat{x}-x_{1}\right)\left(\hat{x}-x_{1}\right)>0,
$$

from Assumption 1. Thus, the firm sets $\hat{x}$ to be at 1 and we have the monopoly result.

Finally, we must show that the monopoly and duopoly results are sensitive to time only with respect to the entry timing. We show in three steps that this point holds.

First, after the follower enters the market (at a given $T_{2}$ ), the corresponding profits are independent of time $t$. That is, firm 1 maximises the following with respect to $p_{1 t}$ :

$$
\max _{p_{1 t}} \int_{0}^{\bar{x}} p_{1 t}\left(x ; x_{1}, x_{2}, p_{2 t}\right) d x
$$

and firm 2 maximises the following with respect to $p_{2 t}$ :

$$
\max _{p_{2 t}} \int_{\bar{x}}^{1} p_{2 t}\left(x ; x_{1}, x_{2}, p_{1 t}\right) d x .
$$

The result is the aforementioned simple duopoly.

Second, given this result, firm 2 determines $T_{2}$. Firm 2 maximises equation (3) with respect to $T_{2}$, and notably, this process does not depend on how firm 1 sets its prices before firm 2 enters the market.

Third, because firm 1's price before firm 2's entry, $p_{1 t}, t \in\left[0, T_{2}\right)$, does not affect postentry competition (the first step) or entry timing (the second step), firm 1 maximises the following with respect to $p_{1 t}$ :

$$
\max _{p_{1 t}} \int_{0}^{1} p_{1 t}\left(x ; x_{1}\right) d x .
$$

Therefore, the profit maximisation problem corresponds to the maximisation of the integrand. Therefore, firm 1 achieves the monopoly instantaneous profit flow as previously given.

Hence, we show that the maximisation of the instantaneous profit flows is equivalent to the maximisation of total profits. 


\section{References}

Azevedo, Alcino and Dean Paxson. 2014. Developing Real Option Game Models. European Journal of Operational Research, 237(3), pp. 909-920.

Biscaia, Ricardo and Isabel Mota. 2013. Models of Spatial Competition: A Critical Review. Papers in Regional Science, 92(4), pp. 851-871.

Brown, Stephen. 1989. Retail Location Theory: The Legacy of Harold Hotelling. Journal of Retailing, 65(4), pp. 450-470.

Chronopoulos, Michail, Bert De Reyck, and Afzal Siddiqui. 2014. Duopolistic Competition under Risk Aversion and Uncertainty. European Journal of Operational Research, 236(2), pp.643-656.

d'Aspremont, Claude, Jean Jaskold Gabszewicz, and Jacques-Francois Thisse. 1979. On Hotelling's "Stability in Competition". Econometrica, 47(5), pp. 1145-1150.

Dixit, Avinash K. and Robert S. Pindyck. 1994. Investment under Uncertainty. Princeton University Press.

Fudenberg, Drew and Jean Tirole. 1985. Preemption and Rent Equalization in the Adoption of New Technology. The Review of Economic Studies, 52(3), pp. 383-401.

Götz, Georg. 2005. Endogenous Sequential Entry in a Spatial Model Revisited. International Journal of Industrial Organization, 23(3-4), pp. 249-261.

Hotelling, Harold. 1929. Stability in competition. Economic Journal, 39(153), 41-57.

Kalyanaram, Gurumurthy, William T. Robinson, and Glen L. Urban. 1995. Order of Market Entry: Established Empirical Generalizations, Emerging Empirical Generalizations, and Future Research. Marketing Science, 14(3s), pp. G212-G221. 
Kress, Dominik and Erwin Pesch. 2012. Sequential Competitive Location on Networks. European Journal of Operational Research, 217(3), pp. 483-499.

Krishnan, Viswanathan and Karl T. Ulrich. 2001. Product Development Decisions: A Review of the Literature. Management Science, 47(1), pp. 1-21.

Lambertini, Luca. 1997. Unicity of the Equilibrium in the Unconstrained Hotelling Model. Regional Science and Urban Economics, 27(6), pp. 785-798.

Lambertini, Luca. 2002. Equilibrium Locations in a Spatial Model with Sequential Entry in Real Time. Regional Science and Urban Economics, 32(1), pp. 47-58.

Lancaster, Kelvin. 1990. The Economics of Product Variety: A Survey. Marketing Science 9(3), pp. 189-206.

Meza, Sergio and Mihkel Tombak. 2009. Endogenous Location Leadership. International Journal of Industrial Organization, 27(6), pp. 687-707.

Neven, Damien J. 1987. Endogenous Sequential Entry in a Spatial Model. International Journal of Industrial Organization, 5(4), pp. 419-434.

Prescott, Edward C. and Michael Visscher. 1977. Sequential Location among Firms with Foresight. The Bell Journal of Economics, 8(2), pp. 378-393.

Thomadsen, Raphael. 2007. Product Positioning and Competition: The Role of Location in the Fast Food Industry. Marketing Science, 26(6), pp. 792-804.

Vakratsas, Demetrios, Ram C. Rao, and Gurumurthy Kalyanaram. 2003. An Empirical Analysis of Follower Entry Timing Decisions. Marketing Letters, 14(3), pp. 203-216. 